\title{
Laser-Induced Focused Ultrasound for Cavitation Treatment: Toward High-Precision Invisible Sonic Scalpel
}

\author{
Taehwa Lee, Wei Luo, Qiaochu Li, Hakan Demirci, and L. Jay Guo*
}

\begin{abstract}
$\boldsymbol{B}_{\text {eyond the implementation of the photoacoustic effect to photoacoustic imaging and }}$ laser ultrasonics, this study demonstrates a novel application of the photoacoustic effect for high-precision cavitation treatment of tissue using laser-induced focused ultrasound. The focused ultrasound is generated by pulsed optical excitation of an efficient photoacoustic film coated on a concave surface, and its amplitude is high enough to produce controllable microcavitation within the focal region (lateral focus $<100 \mu \mathrm{m})$. Such microcavitation is used to cut or ablate soft tissue in a highly precise manner. This work demonstrates precise cutting of tissue-mimicking gels as well as accurate ablation of gels and animal eye tissues.
\end{abstract}

\section{Introduction}

The photoacoustic (PA) effect (light-to-sound conversion) has been widely used for imaging and sensing applications in medicine and industry due to its noncontact, nondestructive characteristics. In these applications, pulsed (or modulated) light is irradiated on light-absorbing materials, producing PA signals via transient heating followed by the thermal expansion of the materials, and measurement of the PA signals allows to determine certain properties of the materials.

\section{Dr. T. Lee, Prof. L. J. Guo}

Department of Mechanical Engineering

The University of Michigan

Ann Arbor, MI 48109, USA

E-mail: guo@umich.edu

Prof. W. Luo, Q. Li, Prof. L. J. Guo

Electrical Engineering and Computer Science

The University of Michigan

Ann Arbor, MI 48109, USA

Prof. W. Luo

School of Optical and Electrical Information

Huazhong University of Science and Technology

Wuhan, Hubei 430074, China

Prof. H. Demirci

Kellogg Eye Center

The University of Michigan

Ann Arbor, MI 48109, USA

The ORCID identification number(s) for the author(s) of this article can be found under https://doi.org/10.1002/smll.201701555.

DOI: 10.1002/smll.201701555
Equipped with a pulsed laser, PA systems can generate broadband ultrasound pulses, which are useful in high-resolution imaging, e.g., PA imaging for structural, functional, and molecular imaging. ${ }^{[1-4]}$ Beyond PA imaging, new applications of the PA effect have been recently investigated, which include all optical fiber-based ultrasound imaging ${ }^{[5,6]}$ and therapeutic applications using high-amplitude photoacoustic pulses. ${ }^{[7-9]}$ Notably, these applications used PA transmitters consisting of a PA converting film coated on a transparent substrate. ${ }^{[10-13]}$ Similar to piezoelectric transducers, the PA transmitters deliver ultrasound pulses to tissue, whereas in PA imaging tissue itself serves as a sound emitter. Baac and co-workers demonstrated focused PA generation using a PA transmitter composed of a concave optical lens with a PA coating layer made of a carbon nanotube (CNT) composite (referred to as a CNT lens). The focused PA pulses by the CNT lens were strong enough to cause microcavitation bubbles on a glass slide in water. ${ }^{[14]}$ Also, they found that violent growth and collapse of the microbubbles caused targeted mechanical disturbance to cells cultured on glass substrates, ${ }^{[7,15,16]}$ showing the potential to realize high-precision targeted cavitation therapy. However, this approach has a limitation, as the microcavitation was only observed in the presence of the solid surface.

Controlled microcavitation alternatively generated by a pulsed laser via optical breakdown or vaporization, has been studied for a long time and even used for therapeutic applications such as laser surgery of water-rich soft tissue. ${ }^{[17,18]}$ However, such an optical approach for cavitation generation is limited to transparent tissue or superficial tissue due to the limited optical penetration. As an acoustic approach for cavitation generation, histotripsy is free from the limitation, 
as it relies on cavitation induced by focused ultrasound pulses. ${ }^{[19,20]}$ In histotripsy, cavitation mechanically fractionates tissue into acellular homogenate, without causing unwanted thermal damages to the surrounding tissue. The nonthermal, mechanical effects produced by histotripsy allows significantly improved therapy precision, compared to typical high-intensity focused ultrasound (HIFU), which produces heat over a relatively large focal volume $(>\mathrm{mm})$ accompanied by uncontrolled cavitation. ${ }^{[21]}$ To achieve negative pressure amplitudes $\left(P_{-}>20 \mathrm{MPa}\right)$ higher than the cavitation threshold in water, histotripsy requires a relatively large aperture of piezoelectric transducers for a high focal gain, which, however, may have the limited acoustic window when treating deep tissue and thus need further increase in the pressure amplitude. ${ }^{[22]}$ Although a relatively large focal volume produced by histotripsy is effective in treating sizable lesions, it would be challenging to achieve microprecision cavitation treatment.

Regulated by laser-induced focused ultrasound within its tight focal volume $(\approx 100 \mu \mathrm{m})$, acoustic microcavitation has similar advantages to those of histotripsy, but it has higher precision for applications, e.g., cavitation therapy of vital organs such as brain and eyes with small lesions surrounded by vulnerable nerves. Additionally, compact PA transmitters $(<15 \mathrm{~mm}$ ) could allow an easier experimental setup, and can be easily integrated to an endoscopic system. Despite these advantages, the main limitation of microcavitation previously demonstrated through PA lenses was that targets such as cells or tissue had to be placed on a rigid substrate (e.g., glass) that serves as a sound reflector. Without the rigid substrate, the photoacoustic pulses were not found to be strong enough to cause microcavitation, as the rigid substrate induces acoustic reflection and interference, resulting in pressure doubling near the substrate. ${ }^{[14]}$ Such limitation hinders from applying the microcavitation treatment to tissue inside the body, far away from rigid materials.

In this work, we demonstrate high-precision cavitation treatment of water-rich soft materials, which is enabled with a recently-optimized PA lens with improved focal gain, capable of generating free-field microcavitation $(<100 \mu \mathrm{m}){ }^{[23]}$ Two different modes of the cavitation treatment, cutting and ablation, are demonstrated. The cavitation-based sonic cutting is capable of precisely cutting tissue-mimicking gels into primitive shapes $(<1 \mathrm{~mm})$ as well as into complex shapes $(<3 \mathrm{~mm})$, creating a small kerf of $<50 \mu \mathrm{m}$. In addition to the cutting mode, this approach allows for accurately ablating a certain volume of tissue-mimicking gel, and is also applied to tissues such as intestinal cell spheroids (called organoids) and pig eyeball. This modality could open new possibilities in realizing highprecision, noninvasive surgery of small diseased lesions, while protecting normal nerves and tissue near the malignant tissue.

\section{Results and Discussion}

\subsection{High-Precision Cutting of Tissue-Mimicking Gel}

Figure 1a shows the schematics of a cavitation treatment setup consisting of a laser-induced focused ultrasound system and a three-axis motorized stage. A pulsed laser beam (pulse duration: $6 \mathrm{~ns}$, wavelength: $532 \mathrm{~nm}$ ) was irradiated onto a thin CNT composite film coated on the concave side of a plano-concave optical lens, producing photoacoustic pulses that converge at the center of the curvature of the concave surface (Diameter $D=15 \mathrm{~mm}$, radius of curvature $r=f=9.2 \mathrm{~mm}$, and $f$-number $=r / D=0.61)$. The focused photoacoustic pulses (center frequency $f_{\mathrm{c}}=15 \mathrm{MHz}$ ) are strong enough to create free-field microcavitation in water (negative pressure amplitude $P_{-}>30 \mathrm{MPa}$ ), without the necessity to put a sound-reflecting rigid material near the focus. ${ }^{[23]}$ The microcavitation was very controllable, as it can be regulated spatially within the focal volume and temporally through the ultrasound pulses. Such controlled microcavitation is capable of localizing mechanical effects (disruption), allowing microcutting of water-based materials such as agarose gel. In the experiment, both the laser beam and PA lens were fixed and thus cavitation remained at a certain location, while samples were precisely moved along predefined paths through the motorized stage with a micrometer resolution.

Figure $1 \mathrm{~b}$ illustrates the schematics of cavitation-based ultrasonic cutting for a penetrating square hole $\left(x \times y=1 \mathrm{~mm}^{2}\right)$. A thin tissue-mimicking gel of $1 \mathrm{~mm}$ thickness was sequentially cut by creating microcavitation on the four imaginary surfaces enclosing a square, with step sizes of $\Delta x$ (or $\Delta y)=50 \mu \mathrm{m}$ and $\Delta z=50 \mu \mathrm{m}$. Note that the $z$-depth of the cutting surfaces ( $3 \mathrm{~mm})$ is larger than the gel thickness to ensure a complete cutout. The cavitation cutting was conducted at a laser pulse energy of $16 \mathrm{~mJ}$ pulse $^{-1}$, which corresponds to a negative pressure amplitude of $P_{-}=30 \mathrm{MPa}$ at the focus. The applied negative pressure amplitude is higher than the bubble nucleation threshold $\approx 24 \mathrm{MPa}$ (the nucleation threshold is defined as the lowest negative pressure amplitude that has a cavitation probability of 1$)$. The cube $\left(1 \times 1 \times 1 \mathrm{~mm}^{3}\right)$ and hole are shown in Figure 1b. Also, the other primitive shapes (circle and triangle) were easily produced by the cavitation cutting (Figure 1c), all exhibiting sharp cut edges.

To demonstrate the capability of the high-precision cavitation cutting applicable to more complex shapes beyond primitive shapes, we performed cavitation cutting for shapes in the forms of the University of Michigan (M) logo and the US Mainland, as shown in Figure 2c,f. The cavitation cutting was conducted along the predefined paths that were extracted and digitized from images of the logo and map, and were scaled to a width of $3 \mathrm{~mm}$, as shown in Figure 2a,d.

Photographs of the as-cut gel samples are shown in Figure 2b,e. In Figure 2b, the straight lines carved on the thin gel were clearly seen, having a kerf of $\approx 45 \mu \mathrm{m}$. Although the cut inner part was structurally detached from the outside, water surface tension kept them together, requiring a gentle push of the inside part for separation. The $\mathrm{M}$ shape and hole are shown in Figure 2c. As compared to the $M$ shape, the outline of the US map consists of more complex curves, mimicking actual lesions. Both the inner and outer parts were well separated after the cutting, as shown in Figure 2f. Note that the narrow and long region of Florida (only $100 \mu \mathrm{m}$ in width) was successfully preserved.

To show the advantages of our cutting approach over conventional laser cutting techniques, our cavitation-based 
(a)

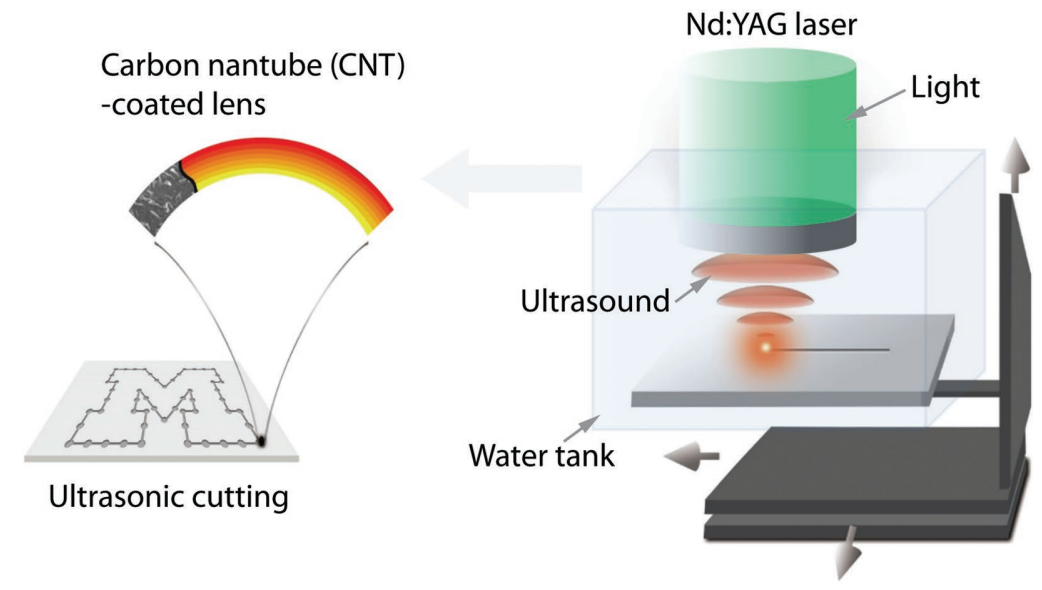

(b)

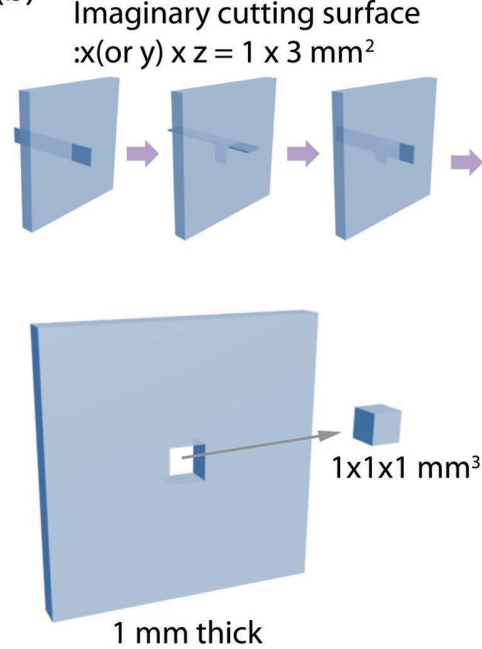

(c)

Figure 1. a) Experimental setup for cavitation-based sonic cutting. Laser-induced focused ultrasound creates microcavitation in a sample mounted on a motorized stage. The sample moves along a predefined path through the motorized stage while the microcavitation remains at a fixed location. b) Schematics of sonic cutting of tissue-mimicking gel ( $1 \mathrm{~mm}$ thick) for a square hole $\left(1 \times 1 \mathrm{~mm}^{2}\right)$. Ultrasonic microcavitation is sequentially applied on four imaginary cutting surfaces $\left[x(\right.$ or $y) \times z=1 \times 3 \mathrm{~mm}^{2}$ ], resulting in the hole upon a removal of the cube $\left(1 \times 1 \times 1 \mathrm{~mm}^{3}\right)$. Photo of the perforated gel and cube was taken after cutting. c) Other primitive shapes (circle and triangle) produced by the sonic cutting.

cutting was applied to incise black gels that does not allow light to penetrate. The M logo was carved on the black gel, shown in Figure 3. The kerf widths at the top and bottom remained the same, indicating the uniform cuts throughout the depth, which cannot be easily achieved by optical approaches typically having $\mathrm{v}$-shaped taper. Although laser cutting methods based on dielectric breakdown show promise in cutting transparent soft tissue, cutting opaque materials by these methods would be significantly limited, especially when the nontransparent materials are buried by opaque materials. This indicates that our cavitation-based cutting approach could be potentially applied to tissues deep inside the body, as long as the laser-generated ultrasound pulses are not significantly attenuated during propagation.

\subsection{Characterization of Microcavitation}

Figure 4 shows shadowgraph images of PA pulses and microcavitation in water (a) and tissue-mimicking gel (b) at a laser pulse energy of $E=18.5 \mathrm{~mJ}$ per pulse (corresponding to $P_{-}=35 \mathrm{MPa}$ ), which is slightly higher than that used in the cutting experiment in order to create larger bubbles for easier observation. In both cases, the visualized PA waves generated from the PA lens, converged at the focus $(t=0.1 \mu \mathrm{s})$. After the PA pulses passed through the focus, bubble clouds were clearly observed within the focal region $(t=0.2 \mu \mathrm{s})$. The bubbles rapidly grew, coalesced, and then collapsed; the bubble lifetimes $\left(t_{1}\right)$ were about $25 \mu$ s in water and $11 \mu \mathrm{s}$ in the gel before final collapse. The shorter lifetime in the gel is because the gel confines the bubble growth, and thus the maximum bubble in the gel was smaller than that in water. Note that the bubble lifetimes are much greater than the negative duration of the PA pulses $(<100 \mathrm{~ns})$. Therefore, the PA pulses strongly influence bubble nucleation and initial growth, while the rest of bubble dynamics in the absence of pressure field is driven by inertia.

Under the strong pressure pulse, tiny bubbles were densely nucleated within the focal volume, which has an elliptical shape elongated along the $z$-axis $\left(l_{r}<l_{z}\right.$, where $l_{r}, l_{z}$ 
(a)

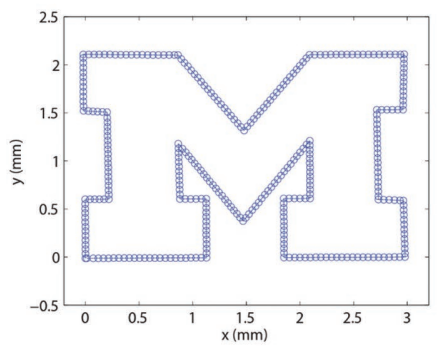

(d)

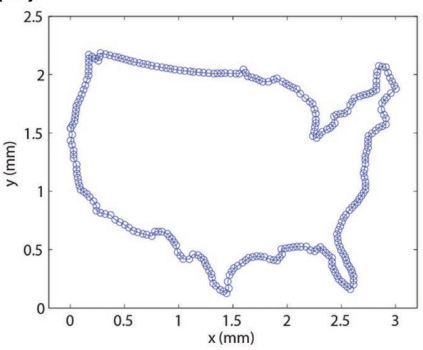

(b)

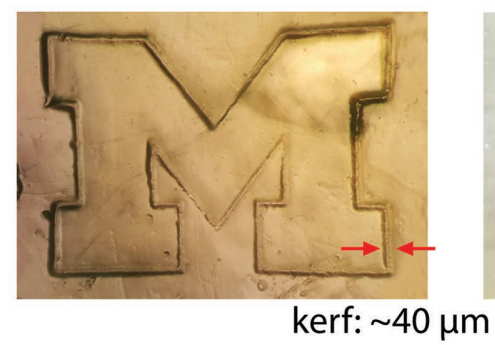

(e)

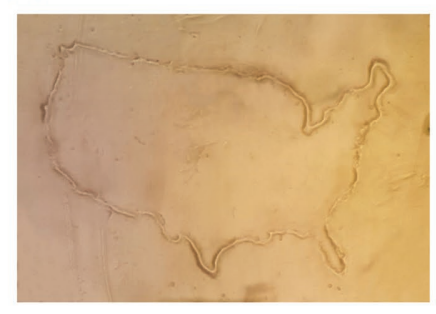

(c)

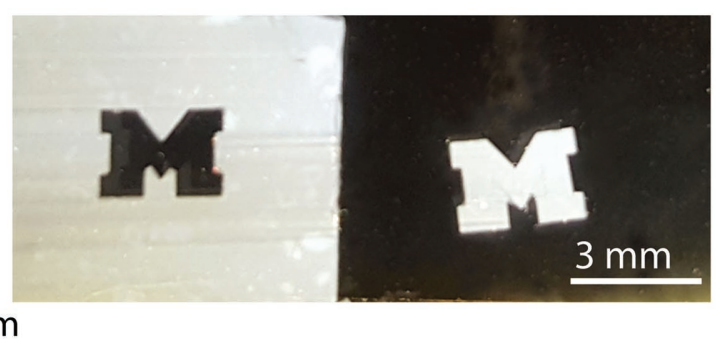

(f)

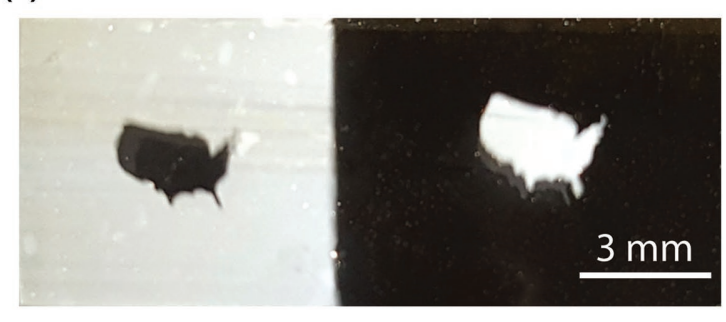

Figure 2. Cavitation-based sonic cutting of tissue-mimicking gel for complex shapes: the logo of University of Michigan, the United States map. a,d) Predefined cutting paths. b,e) Photos of the treated gels before removing the inner parts. The photos were taken by a microscope, looking yellowish. $c, f)$ Photos of the holes and the inner parts. The widths are $3 \mathrm{~mm}$. The photos were taken by a digital camera.

are the $r$-width and $z$-width), as dictated by the focused pressure field as shown in the simulation (Figure 5a). The dark red region $\left(\sigma_{\text {sim }}=l_{r} / l_{z}=0.3\right)$ corresponds to the region with high pressure amplitudes. The elongated pressure field is consistent with the measured pressure profiles in lateral and longitudinal directions (Figure 5c,d). As shown in Figure 5b, the visualized bubble region in water $\left(\sigma_{\mathrm{vis}}=l_{r} / l_{z}=95 \mu \mathrm{m} / 285 \mu \mathrm{m}=\right.$ 0.33 at $t=0.4 \mu \mathrm{s}$ ) shows a good match with the simulated focal region $\left(\sigma_{\text {sim }}=0.3\right)$ since a bubble nucleation region is determined as an area where negative pressure amplitudes are greater than cavitation threshold. The kerf measured in the cutting demonstration $(45 \mu \mathrm{m})$ is much smaller than the largest bubbles in the gel (e.g., $t=6 \mu \mathrm{s}$ ), while it is similar to the lateral width of the bubble nucleation region $(t=0.2 \mu \mathrm{s})$. This finding suggests that explosive bubble growth at the

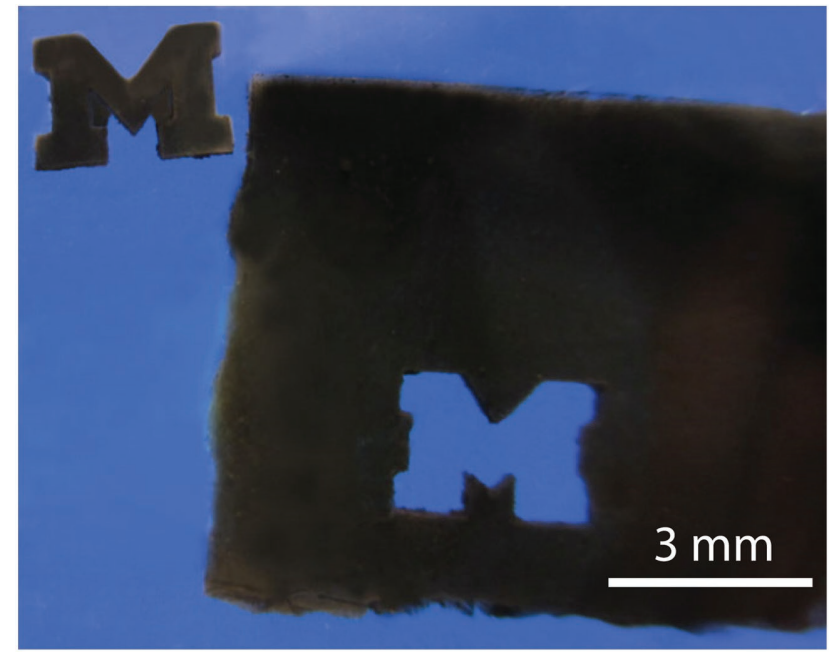

Figure 3. Cavitation-based sonic cutting of black tissue-mimicking gel. early stage after its inception is more destructive than the later bubble growth, and thus is responsible for the cutting.

Figure 6 shows cutting performance by using laser energies ranging from 14 to $18.5 \mathrm{~mJ}$ per pulse. All the cuts, labeled with the numbers from 1 to 4 , were clearly observed (Figure 6a). For the lowest pressure amplitude (but above the cavitation threshold), the inner part cannot be removed from the gel (Figure 6b), because the bubble nucleation region is not large enough to produce continuous cutting, even with step sizes of $\Delta x=\Delta y=50 \mu \mathrm{m}$. With increasing pressure amplitude, the bubble nucleation region increases, and eventually form a well-connected path that completes the cutting.

The number of the tiny bubbles $\left(n_{\mathrm{B}}\right)$ is estimated to be $n_{\mathrm{B}}>30$ based on the shadowgraphy images, corresponding to an average bubble space of $d<20 \mu \mathrm{m}$. Such short interbubble distance can cause strong bubble-bubble interaction as the nondimensional standoff distance $\gamma=d /\left(2 R_{\max }\right)<1$, ${ }^{[24]}$ where $R_{\max }$ is the maximum bubble radius that is calculated for an isolated single bubble $\left(R_{\max }=30 \mu \mathrm{m}\right.$ for $\left.P_{-}=35 \mathrm{MPa}\right)$ by using the Rayleigh-Plesset equation. ${ }^{[25]}$ In the strong interaction regime, expansion of the individual bubbles is significantly restricted, leading to the maximum radius of the merged bubbles $\left(R_{\text {max }^{*}}=200 \mu \mathrm{m}\right.$ in water), which is much smaller than the estimation based on $n_{\mathrm{B}} R_{\max }$, i.e., $R_{\text {max* }}<n_{\mathrm{B}} R_{\max }=900 \mu \mathrm{m}$.

Most of the tiny growing bubbles coalesced into larger bubbles. However, it is interesting to note that in water, some of the bubbles nucleated in the periphery of the cloud were observed to leave from the focal region (see arrows in the images), preferentially moving up due to the buoyancy. This is because violent bubble growth can lead to bouncing bubbles without causing rupture of the liquid film between the bubbles instead of merging bubbles. It is well known that two bubbles bounce rather than 
(a)

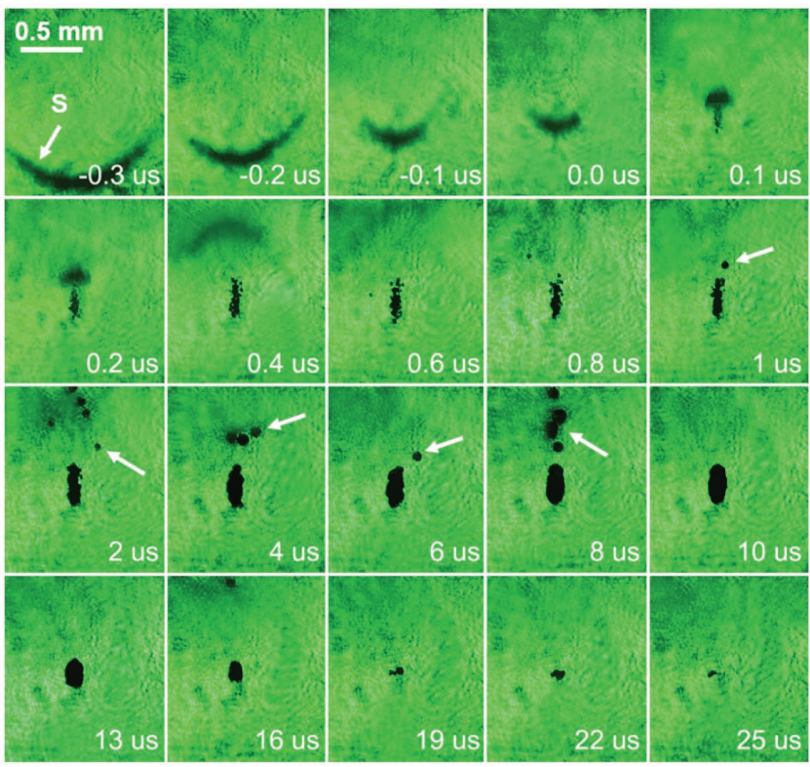

(b)

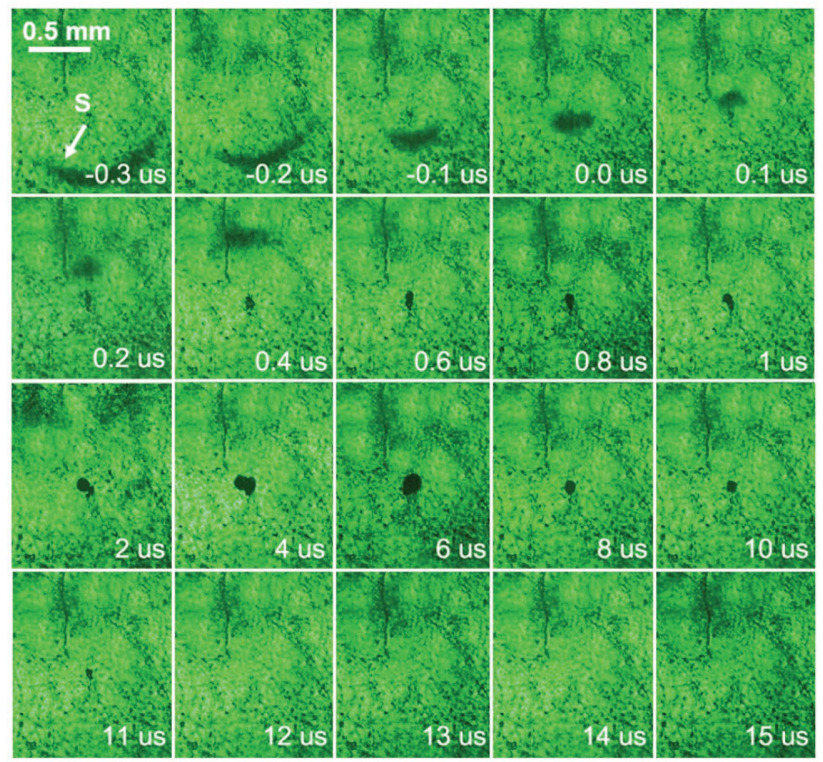

Figure 4. Shadowgraph images of microcavitation in water a) and gel b).

(a)

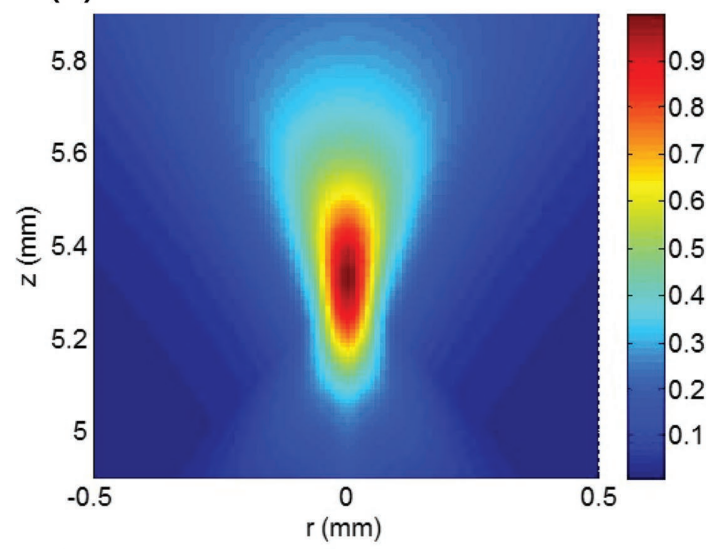

(c)

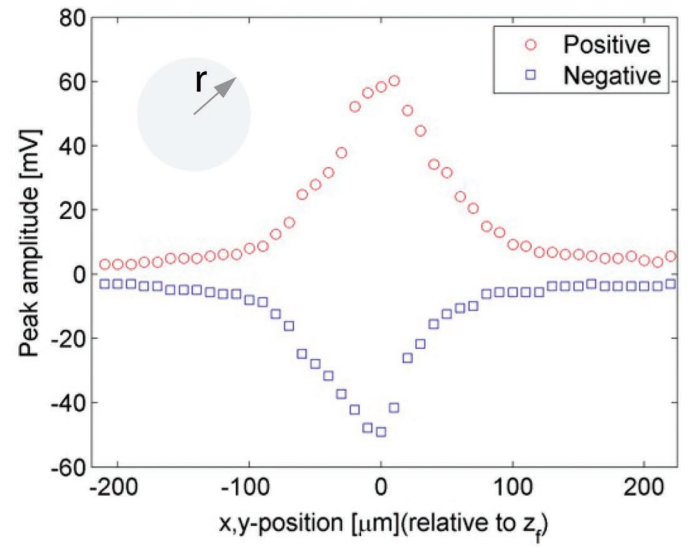

(b)

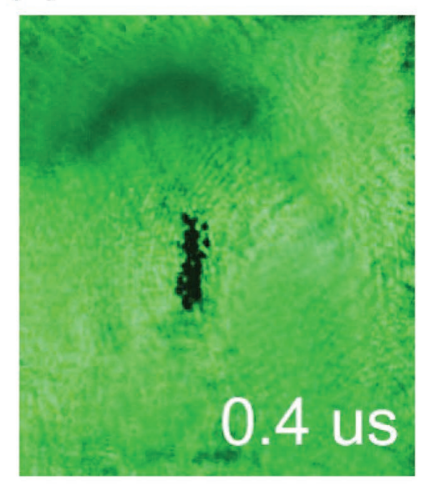

(d)

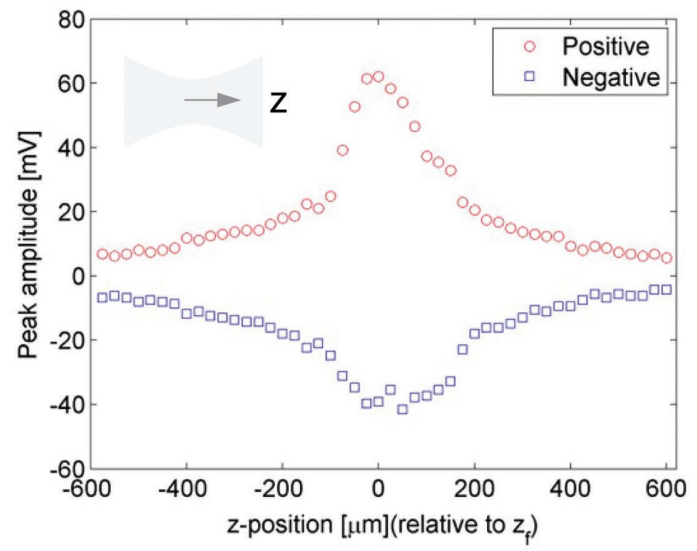

Figure 5. Characterization of focused photoacoustic pulses. a) The simulated focal region. b) Shadowgraph image of bubble nucleation region $(t=0.4 \mu \mathrm{s})$. Focal beam width in $r$-direction $c)$ and $z$-direction $\mathrm{d})$. 
(a)

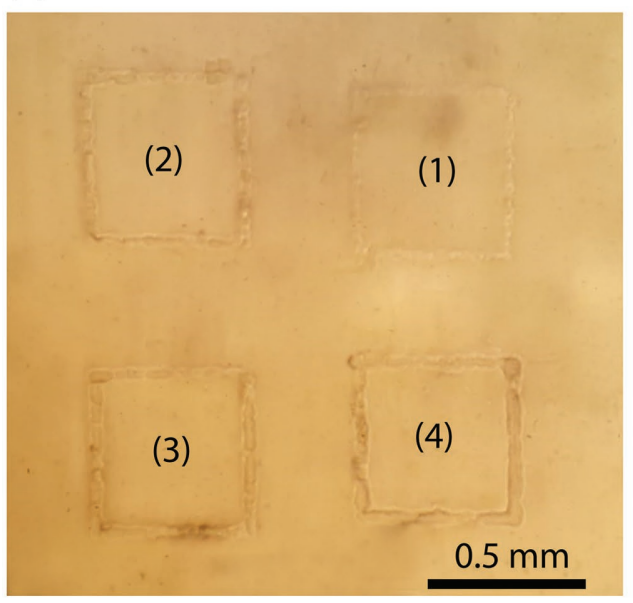

(b)

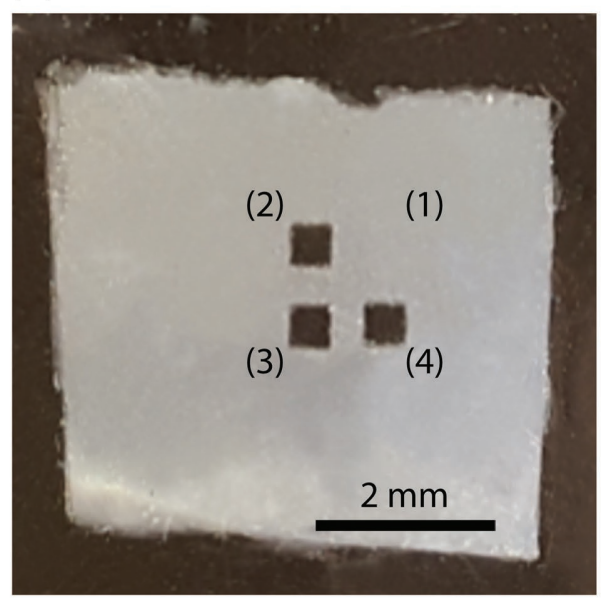

Figure 6. Cavitation-based sonic cutting at different laser pulse energies $(E=14,16,17.5,18.5 \mathrm{~m}$ J per pulse from (1) to (4)). a) Photo of the treated gel having four squares before removing the inner parts. b) Photo after removing the inner parts. For the lowest energy, the inner part cannot be removed.

coalesce if their relative approach velocity is sufficiently high (i.e., high Weber number, $\left.W_{\mathrm{e}}=\rho V^{2} R_{\max } / \sigma>10\right) .{ }^{[26]}$ The individual bubbles that are closely packed will rapidly expand due to the strong pressure pulse, thus falling into the bouncing regime (estimated bubble growth speed of $\left.V \approx 150 \mathrm{~m} \mathrm{~s}^{-1}, W_{\mathrm{e}}>10^{3}\right)$. However, only the outer bubbles can escape from the cloud, whereas the inner bubbles surrounded by the outer bubbles remain in contact with the surrounding bubbles, resulting in their coalescence. On the other hand, no bubble departure was observed in gel; the bubbles stayed within the focal region, merging into larger bubbles due to gel confinement.

\subsection{Volumetric Ablation of Tissue-Mimicking Gel}

Our cavitation-based sonic cutting approach shows promise in high-precision microcutting and the potential to realize microsurgery of diseased tissue. Not only can diseased tissues be cut out from healthy surroundings, but also they can be mechanically ablated for treatment of the entire volume of the tissue. Cells constituting tissue can be fractionated to acellular homogenates by localized mechanical effects associated with microcavitation, which is similar to histotripsy. ${ }^{[20]}$ The cavitation-based ablation approach is illustrated in Figure 7a. A chunk of transparent gel $\left(5 \times 5 \times 10 \mathrm{~mm}^{3}\right)$ was partially ablated through a scanning volume of $0.5 \times 0.5 \times 3 \mathrm{~mm}^{3}$ (colored purple) at a laser pulse energy of $E=17.5 \mathrm{~mJ}$ per pulse; microcavitation was applied on $x-y$ planes $(\Delta x, \Delta y=50 \mu \mathrm{m})$, while moving along the $z$ axis with a spacing of $50 \mu \mathrm{m}$. For easy alignment, the $z$ length of the cavitation scanning volume $(3 \mathrm{~mm})$ is much greater than the actual depth of the ablation volume. As shown in Figure $7 b$, the cavitation-based ablation was conducted for square trenches on the surface of the gel block under conditions of two different intervals $(\Delta x, \Delta y, \Delta z=50$ or $100 \mu \mathrm{m})$. For the large interval $(100 \mu \mathrm{m})$, the ablation effect was not significant, only showing numerous dark spots separated apart on the gel surface. This result indicates that the spacing is too large to form a continuous treatment volume. However, for the small interval $(50 \mu \mathrm{m})$, the deep trench was successfully produced on the gel block, whose depth corresponds to the overlap between the cavitation scanning volume and the gel block.

\subsection{Application to Tissues}

\subsubsection{Intestinal Cell Spheroids (Called Organoids)}

To demonstrate the capability of the cavitation-based treatment of tissue, we used intestinal cell spheroids (called organoids), which are a 3D organ-bud grown in vitro that shows realistic microanatomy. The human intestinal organoids are believed to be the stimulated microhuman organs which has more similarity than the other $3 \mathrm{D}$ structures. ${ }^{[27,28]}$ Cell spheroids with a volume of $\approx 1 \mathrm{~mm}^{3}$ are fixed in metrigel and immersed in $20 \%$ Fetal Bovine Serum cell culture media, and then located right at the focus of the PA lens. The spheroids were scanned by a $3 \mathrm{D}$ stage for a whole treatment volume of $0.8 \times 0.8 \times 1.7 \mathrm{~mm}^{3}(\Delta x, \Delta y, \Delta z=50 \mu \mathrm{m})$; at a laser pulse energy of $E=17.5 \mathrm{~mJ}$ per pulse). The treated sample and a control sample were then imaged by a fluorescent microscope (Olympus BX-51). As seen in Figure 8, the ablated spheroids (dashed square mark) were clearly observed.

\subsubsection{Pig Eyeball}

The pig eye is similar in size and structure to human eye and contains vitreous humor, a water-based gel. It also has a thick outer layer that is called sclera. This thick outer layer can affect the acoustic propagation and thus therapeutic outcomes. Using a pig eye to create cavitation ablation can provide information that could be applicable to human eyes. To demonstrate this effect, we cut the pig eye into a piece of $5 \times 5 \mathrm{~mm}^{2}$ for easy visual confirmation of therapeutic effects 
(a)
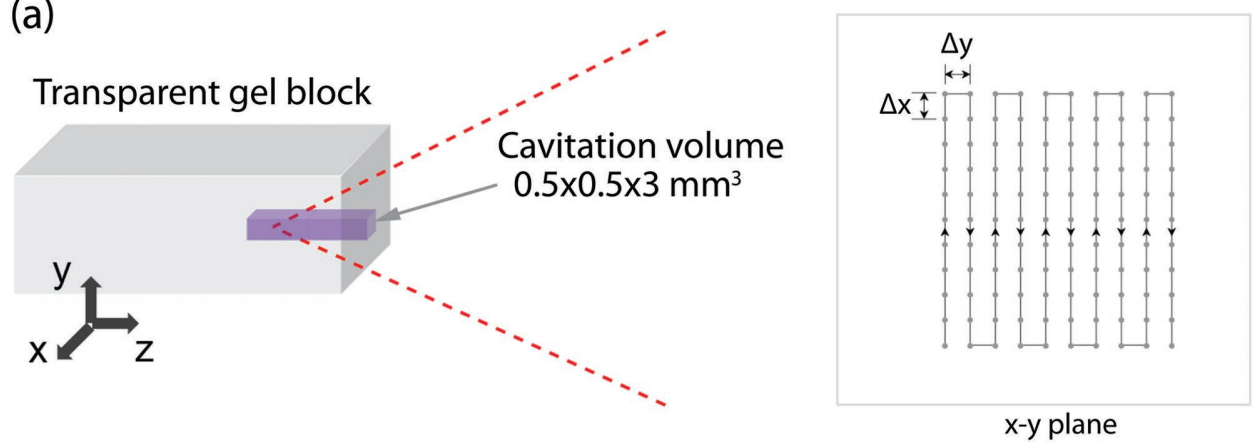

(b)

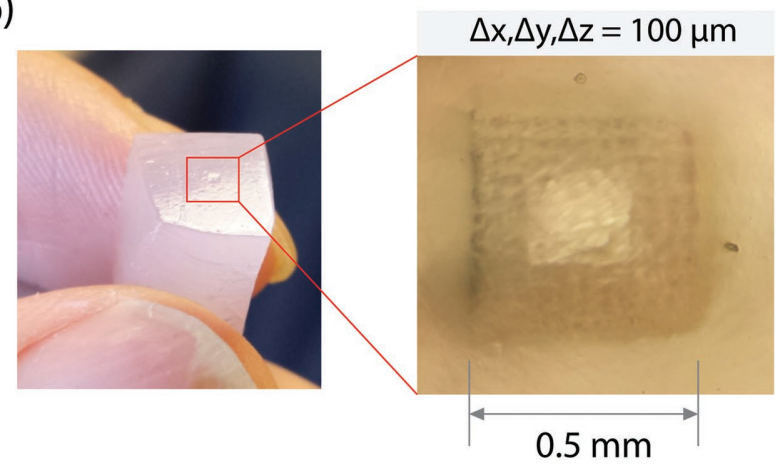

Microscope image $(x 5)$

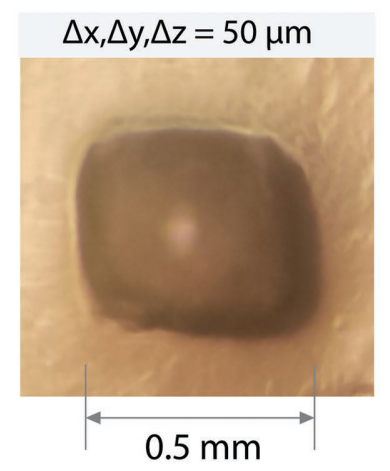

Figure 7. Cavitation-based volumetric ablation. a) Illustration of the volume ablation (purple) of gel block. The cavitation volume of $0.5 \times 0.5 \times 3 \mathrm{~mm}{ }^{3}$ is formed by ablating $x-y$ planes $(\Delta x, \Delta y=50 \mu \mathrm{m})$ at each $z$ location $(\Delta z=50 \mu \mathrm{m})$. b) Photos of the treated gel for two different intervals $(\Delta=50,100 \mu \mathrm{m})$.

and for easy mounting to the cavitation ablation system, as shown in Figure 9a. The piece contains a thick scleral outer layer, vascular choroidal layer and retinal nerve layer. To investigate the effect of the thick scleral outer layer on PA propagation, two different settings were used, as shown in Figure 9b,c. First, PA pulses were directly focused on the vascular dark-colored choroid without passing through the thick sclera (Figure 9b). In the other setting, the piece was

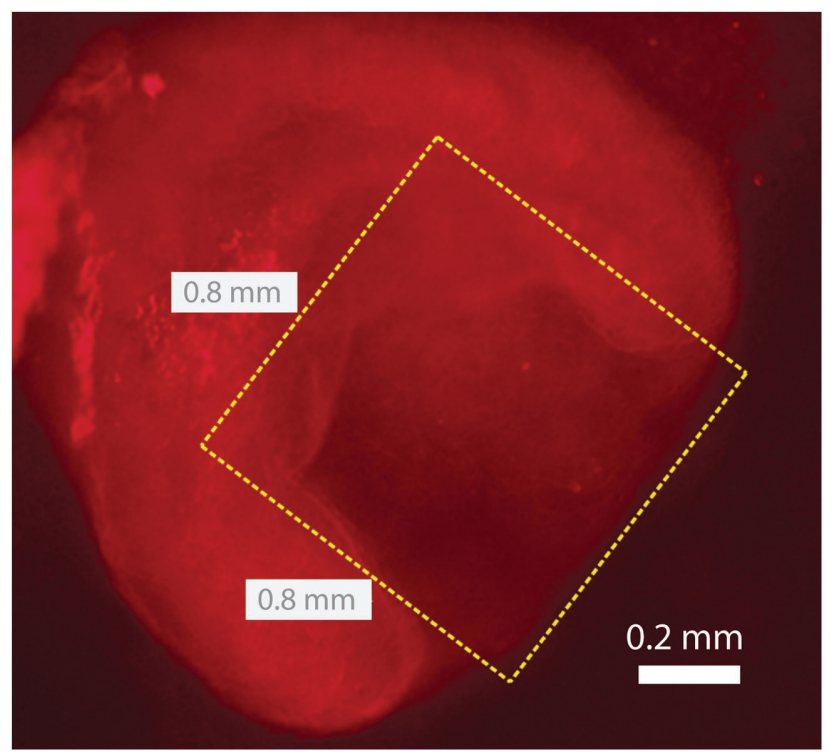

Figure 8. Cavitation-based volumetric ablation of Organoid. flipped so that PA pulses passed through the sclera and were focused on the choroid (Figure 9c). The cavitation ablation qs conducted for two square trenches spaced with $0.2 \mathrm{~mm}$, each trench of $0.5 \times 0.5 \mathrm{~mm}^{2}$. For both settings, mechanical ablation was visually confirmed, showing two small ablated trenches on the choroid after drying the samples. However, the sclera layer caused the acoustic attenuation when PA pulses pass through it, thus requiring compensation by applying slightly higher laser pulse energy ( $E=20 \mathrm{~mJ}$ per pulse).

\section{Conclusions}

We have demonstrated the cavitation-based sonic scalpel by using laser-induced focused ultrasound. Owing to microcavitation regulated by the laser-induced focused ultrasound, such sonic scalpel can accurately cut or ablate water-rich materials such as tissue-mimicking gel and tissues in a targeted, high-precision manner. The resolution of our sonic cutting was $\approx 50 \mu \mathrm{m}$, as confirmed with the kerf after the cutting experiment. Since the sonic scalpel is based on nonthermal cavitation produced by short PA pulses $(\approx 50 \mathrm{~ns})$, thermal effects are negligible. Temperature increase by the short pulses is conservatively estimated to be $10^{-2}{ }^{\circ} \mathrm{C}$ (see the Supporting Information). Our cavitation-based sonic scalpel could be applicable to targeted, noninvasive surgery, where one can treat diseased tissues while minimizing collateral damages to healthy surrounding tissues. 
(a)
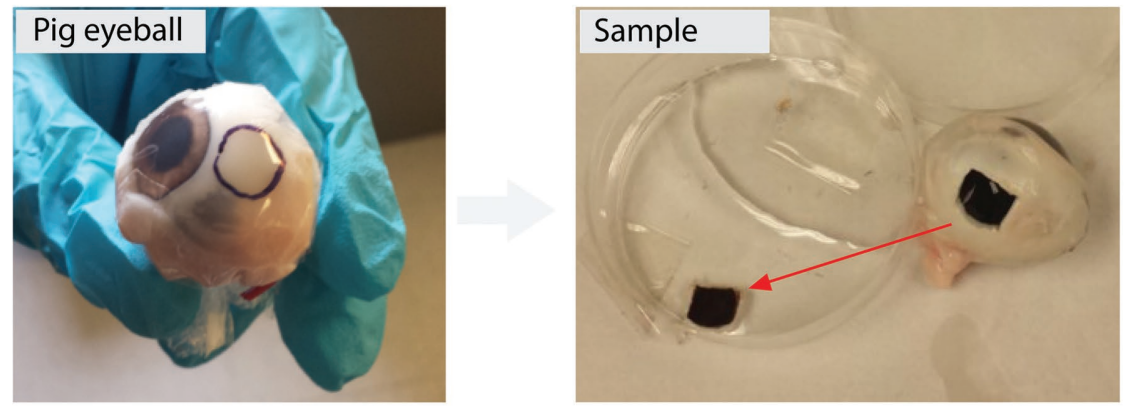

(b)
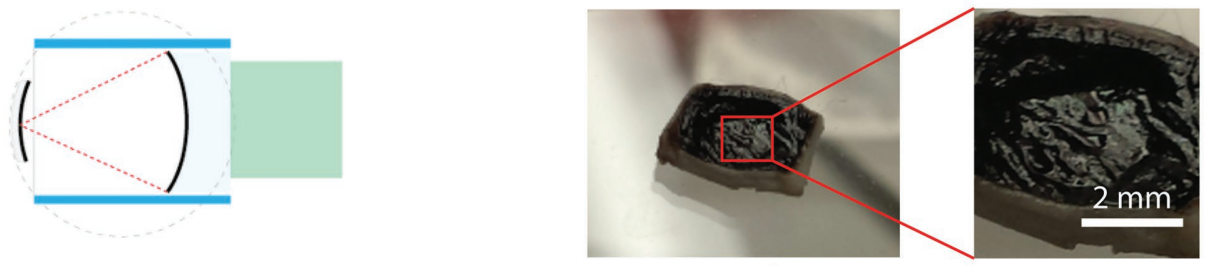

(c)

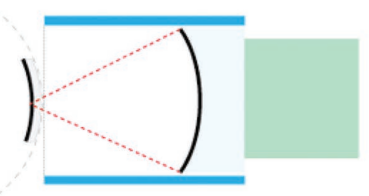

$\mathrm{E}=17.5 \mathrm{~mJ} /$ pulse

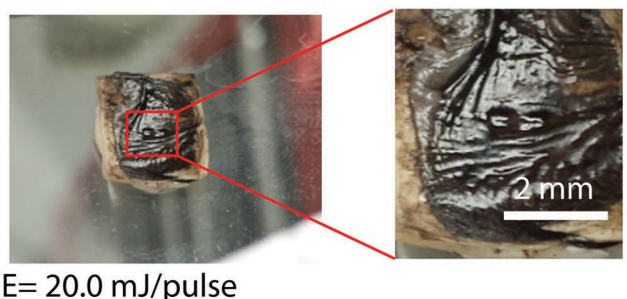

Figure 9. Cavitation-based volumetric ablation of pig eyeball. a) Pig eyeball and the piece of the eyeball with a black Choroid layer and thick white sclera layer. Photoacoustic pulses directly focused on the Choroid layer with c) and without passing through the sclera layer b).

Recent advance in imaging modalities has allowed to accurately delineate margins of lesions with improved imaging resolution. Combined with these imaging modalities, our high-precision sonic scalpel will be effective in treating early-diagnosed small lesions or lesions close to nerves. Specifically, our approach can be implemented for prevision, targeted surgery of brain, because inaccurate treatment can cause brain damage. By isolating diseased lesions (e.g., tumor) in the brain from the surrounding through the cavitation treatment, the diseased tissues are likely to be killed over time. This is because except for some rare cases, brain cells barely regenerate or regenerate slowly, and thus the isolated regions cannot get sufficient blood supply and nutrients.

Our cavitation-based sonic treatment can be readily customized by optimizing ultrasound parameters depending on surgical applications. By tuning laser pulse duration and photoacoustic coating layers, ultrasound frequency can be adjusted in a way that potential acoustic attenuation is minimized when targets are located deep in the body. Because the center frequency of the PA pulses is $\approx 15 \mathrm{MHz}$, pressure amplitudes decrease by $60 \%$ for $5 \mathrm{~mm}$ propagation in tissues (e.g., attenuation coefficient of $1 \mathrm{~dB} \mathrm{~cm}^{-1} \mathrm{MHz}^{-1}$ for kidney). Also, the geometry of PA lenses can be chosen to have a proper focal length and $f$-number for improved cavitation performance. Moreover, the time duration required for the cavitation treatment can be reduced by using a pulsed laser with a higher pulse repetition rate.

\section{Experimental Section}

Laser-Generated Focused Ultrasound: Photoacoustic pulses are generated by irradiating a pulsed laser beam (Nd:YAG laser, pulse duration $=6 \mathrm{~ns}$ full width at half maximum, wavelength $=532 \mathrm{~nm}$ ) onto a CNT-polydimethylsiloxane (PDMS) composite coated on the concave side of a plano-concave fused silica lens (purchased from Optosigma, Santa Ana, CA) with $9.2 \mathrm{~mm}$ radius of curvature and $15 \mathrm{~mm}$ diameter. The PA waves generated by pulsed optical excitation were geometrically focused on the center of the concave. The focused PA pulses generated by the PA lens have a pulse duration of $\approx 50 \mathrm{~ns}$, negative pressure amplitudes of $>30 \mathrm{MPa}$, and the center frequency of $\approx 15 \mathrm{MHz}$ (see Figure $\mathrm{S} 1$ in the Supporting Information). Further details on characterization of the focused PA pulses can be found in the previous paper. ${ }^{[23]}$ To fabricate the CNTPDMS composite-coated lens, ${ }^{[7,10]}$ catalyst layers of Fe $(\approx 1 \mathrm{~nm})$ and Al2O3 $\approx 3 \mathrm{~nm})$ were first coated on the concave surface of the plano-concave lens. Then, multiwalled CNTs were grown on the concave surface in a mixture of $\mathrm{C}_{2} \mathrm{H}_{4} / \mathrm{H}_{2} / \mathrm{He}$ in an atmospheric pressure tube furnace at $770{ }^{\circ} \mathrm{C}$. PDMS elastomer was then spincoated on the CNT-grown surface at $3000 \mathrm{rpm}$ for $1 \mathrm{~min}$, and then cured at $100{ }^{\circ} \mathrm{C}$ for $1 \mathrm{~h}$. The CNT-PDMS composite consists of CNTs 
embedded in PDMS. The CNT composite serves as an excellent light-to-sound converter, because the CNTs efficiently absorb an incident optical energy ( $>80 \%$ ) and convert it to heat that is rapidly transferred to the surrounding PDMS with a high thermal expansion coefficient.

Laser Flash Shadowgraphy: Laser-induced acoustic waves and bubbles were visualized by laser-flash shadowgraphy, a pumpprobe method that allows a probe laser beam (Diode-pumped solid-state laser, FWHM = $5 \mathrm{~ns}, 527 \mathrm{~nm}$, Laser-export Co. Ltd) to cast a shadow of bubbles induced by a pump beam ( $\mathrm{Nd}$ :YAG laser) at a different instance. The temporal moment is specified by a time delay between the pump and probe pulses through a delay generator (Stanford Research Systems, DG535). The shadowgraphs were captured with a CCD camera with a very slow shutter speed $(\approx \mathrm{ms})$, but a short exposure time of $5 \mathrm{~ns}$, determined by the pulse duration of the probe laser, enables for blur-free imaging of fastmoving objects.

Preparation of Tissue-Mimicking Gel: Agarose phantoms were used to provide a well-controlled elastic medium for this study. Agarose phantoms of $1 \% \mathrm{w} / \mathrm{v}$ were prepared by slowly mixing agarose powder (Agarose Type VII, Sigma-Aldrich) into pure water heated at $95{ }^{\circ} \mathrm{C}$. The solution was stirred on a hot plate until the gel turned completely transparent. Agarose solutions were degassed under a partial vacuum of $1 \mathrm{kPa}$ for $30 \mathrm{~min}$ and then was poured into $7.5 \times 2.5 \times 0.1 \mathrm{~cm}$ rectangular glass tissue phantom holders. The tissue phantom holders were placed in a refrigerator at $4{ }^{\circ} \mathrm{C}$ to allow the agarose solution to solidify before use.

Preparation of Intestinal Organoids and Imaging Method: First, pluripotent stem cells were treated with activin A for $3 \mathrm{~d}$ and were differentiated into FOXA2 ${ }^{+} \mathrm{SOSOX} 17^{+}$endoderm. Then, a patterning step was performed to the pluripotent stem cells using FGF4 and WNTNT3a. They were patterned into CDX2 + mid- and hindgut tissue and 3D mid- or hindgut spheroids bud from the monolayer epithelium attached to the tissue culture dish could be found after this step. After that, these 3D spheroids with prointestinal growth factors are further cultured in Matrigel. The 3D spheroids proliferate and expand over several days to give rise to intestinal organoids with suitable sizes. ${ }^{[29]}$ The organoid sample was stained by EthD-1 and Calcein AM [Thermo fisher live/dead cell viability kit] with the volume of 20 and $5 \mu \mathrm{L}$, respectively in $10 \mathrm{~mL}$ hanks solution in petri dish for $30 \mathrm{~min}$. Then the sample was imaged with fluorescent microscope[BX-51] under illumination light of 510-550 nm wavelength for dead cell tracking. However, this method was just used to keep track of the morphology or contour of the organoids instead of differentiating live and dead cells carefully, because the cells in the treated region is depleted rather than simply killed.

Preparation of Pig Eyeball: Pig eyes were harvested by adhering to all standards set forth in the ARVO Statement for the Use of Animals in Ophthalmic and Visual Research. After the eye was enucleated, a $5 \times 5 \mathrm{~mm}$ piece of pig eye was cut from the posterior pole of the globe and treated.

Pressure Field Simulation: To calculate pressure field around the focus, the linear wave equation in a lossless medium is solved by using a finite element method (Courant number $<0.05$; COMSOL Multiphysics $4.3 \mathrm{~b}$ ). For simplicity, the nonlinear wave propagation and attenuation are assumed to be negligible, because it was focused on the acoustic propagation in a small region near the focus $(<1 \mathrm{~mm})$. Instead, a proper incident pressure on the optoacoustic lens is chosen to match the measured signal at the focus. The simulated pressure field around the focus is obtained with the maximum negative pressure amplitude encountered at each location during the simulation.

\section{Supporting Information}

Supporting Information is available from the Wiley Online Library or from the author.

\section{Acknowledgements}

T.L. and W.L. contributed equally to this work. This work was supported by MCube program at the University of Michigan. The authors acknowledge Dr. Shuichi Takayama and Nick Ginga for providing the intestinal organoids for the testing.

\section{Conflict of Interest}

The authors declare no conflict of interest.

[1] M. Xu, L. V. Wang, Rev. Sci. Instrum. 2006, 77, 041101.

[2] A. de la Zerda, J.-W. Kim, E. I. Galanzha, S. S. Gambhir, V. P. Zharov, Contrast Media Mol. Imaging 2011, 6, 346.

[3] K. Pu, A. J. Shuhendler, J. V. Jokerst, J. Mei, S. S. Gambhir, Z. Bao, J. Rao, Nat. Nanotechnol. 2014, 9, 233.

[4] C. Kim, C. Favazza, L. V. Wang, Chem. Rev. 2010, 110, 2756.

[5] R. J. Colchester, E. Z. Zhang, C. A. Mosse, P. C. Beard, I. Papakonstantinou, A. E. Desjardins, Biomed. Opt. Express 2015, 6, 1502.

[6] R. J. Colchester, C. A. Mosse, D. S. Bhachu, J. C. Bear, C. J. Carmalt, I. P. Parkin, B. E. Treeby, I. Papakonstantinou, A. E. Desjardins, Appl. Phys. Lett. 2014, 104, 173502.

[7] H. W. Baac, J. G. Ok, A. Maxwell, K.-T. Lee, Y.-C. Chen, A. J. Hart, Z. Xu, E. Yoon, L. J. Guo, Sci. Rep. 2012, 2, 989.

[8] J. Di, J. Kim, Q. Y. Hu, X. N. Jiang, Z. Gu, J. Controlled Release 2015, $220,592$.

[9] T. Lee, H. W. Baac, J. G. Ok, H. S. Youn, L. J. Guo, Phys. Rev. Appl. 2015, 3, 044007.

[10] H. W. Baac, J. G. Ok, H. J. Park, T. Ling, S.-L. Chen, A. J. Hart, L. J. Guo, Appl. Phys. Lett. 2010, 97, 234104.

[11] W. Y. Chang, W. B. Huang, J. Kim, S. B. Li, X. N. Jiang, Appl. Phys. Lett. 2015, 107, 161903.

[12] R. J. Colchester, C. A. Mosse, D. S. Bhachu, J. C. Bear, C. J. Carmalt, I. P. Parkin, B. E. Treeby, I. Papakonstantinou, A. E. Desjardins, Appl. Phys. Lett. 2014, 104, 173502.

[13] B.-Y. Hsieh, J. Kim, J. Zhu, S. Li, X. Zhang, X. Jiang, Appl. Phys. Lett. 2015, 106, 021902.

[14] T. Lee, H. W. Baac, J. G. Ok, H. S. Youn, L. J. Guo, Phys. Rev. Appl. 2014, 2, 024007.

[15] H. W. Baac, J. Frampton, J. G. Ok, S. Takayama, L. J. Guo, J. Biophotonics 2013, 6, 905.

[16] H. W. Baac, T. Lee, L. J. Guo, Biomed. Opt. Express 2013, 4, 1442.

[17] I. Toytman, A. Silbergleit, D. Simanovski, D. Palanker, Phys. Rev. E 2010, 82, 046313.

[18] A. K. Jayasinghe, J. Rohner, M. S. Hutson, Biomed. Opt. Express 2011, 2, 2590. 
[19] W. W. Roberts, T. L. Hall, K. Ives, J. S. Wolf, J. B. Fowlkes, C. A. Cain, J. Urol. 2006, 175, 734.

[20] Z. Xu, T. L. Hall, J. B. Fowlkes, C. A. Cain, J. Acoust. Soc. Am. 2007, $122,229$.

[21] T. J. Dubinsky, C. Cuevas, M. K. Dighe, O. Kolokythas, J. H. Hwang, Am. J. Roentgenol. 2008, 190, 191.

[22] E. Vlaisavljevich, O. Aydin, K.-W. Lin, Y. Yuksel Durmaz, B. Fowlkes, M. ElSayed, Z. Xu, Phys. Med. Biol. 2015, 61, 663.

[23] T. Lee, J. G. Ok, L. J. Guo, H. W. Baac, Appl. Phys. Lett. 2016, 108, 104102.

[24] I. van der Kroon, P. A. Quinto-Su, F. F. Li, C.-D. Ohl, Phys. Rev. E 2010, 82, 066311.
[25] C. E. Brennen, Cavitation and Bubble Dynamics, Oxford University Press, Oxford, UK 1995.

[26] P. C. Duineveld, Appl. Sci. Res. 1998, 58, 409.

[27] J. R. Spence, C. N. Mayhew, S. A. Rankin, M. F. Kuhar, J. E. Vallance, K. Tolle, E. E. Hoskins, V. V. Kalinichenko, S. I. Wells, A. M. Zorn, N. F. Shroyer, J. M. Wells, Nature 2010, 470, 105.

[28] C. Xinaris, V. Brizi, G. Remuzzi, Nephron 2015, 130, 191.

[29] K. W. McCracken, J. C. Howell, J. M. Wells, J. R. Spence, Nat. Protoc. 2011, 6, 1920.

Received: May 11, 2017

Revised: June 18, 2017

Published online: August 15, 2017 